\title{
Erratum to: Do protected areas in urban and rural landscapes differ in species diversity?
}

\author{
Sonja Knapp • Ingolf Kühn • Volker Mosbrugger • Stefan Klotz
}

Published online: 3 August 2010

(C) Springer Science+Business Media B.V. 2010

\section{Erratum to: Biodivers Conserv DOI 10.1007/s10531-008-9369-5}

To compare species spatial turnover in urban and rural protected areas, we calculated turnover from presence-absence tables for each pair of protected areas (i) within the city of Halle, i.e. urban protected areas, (ii) within the district of Saalkreis that surrounds Halle, i.e. rural protected areas, and (iii) for each pair of urban and rural protected areas. We stated that we used the $\beta_{\text {sim }}$ similarity index as given in Lennon et al. (2001) and Koleff et al. (2003):

$$
\beta_{\text {sim }}=a /(a+\min (b, c))
$$

The function in $\mathrm{R}$ ( $\mathrm{R}$ Development Core Team, 2004) used to calculate $\beta_{\text {sim }}$ was a modified version of dist.binary from the package ade4 (Chessel et al. 2004). However, what this function calculates is not

The online version of the original article can be found under doi:10.1007/s10531-008-9369-5.

S. Knapp $(\bowtie) \cdot$ I. Kühn $\cdot$ S. Klotz

Department of Community Ecology (BZF), UFZ, Helmholtz Centre for Environmental Research, Theodor-Lieser-Str. 4, 06120 Halle, Germany

e-mail: sonja.knapp@ufz.de

I. Kühn

e-mail: ingolf.kuehn@ufz.de

S. Klotz

e-mail: stefan.klotz@ufz.de

S. Knapp · V. Mosbrugger

Institute for Geosciences, University of Tübingen, Sigwartstr. 10, 72076 Tübingen, Germany

e-mail: volker.mosbrugger@senckenberg.de

Present Address:

V. Mosbrugger

Senckenberg Research Institute and Natural History Museum,

Senckenberganlage 25, 60325 Frankfurt, Germany 


$$
\beta_{\text {sim }}=a /(a+\min (b, c))
$$

but

$$
\beta_{\text {dissim }}=\operatorname{sqrt}\left(1-\beta_{\text {sim }}\right)=\operatorname{sqrt}(1-(a /(a+\min (b, c)))) .
$$

This is a measure of dissimilarity ranging from zero to one, the upper limit indicating complete dissimilarity of communities and the lower limit indicating complete similarity. As we mixed-up the similarity index with its derived dissimilarity index, the interpretation of species turnover we gave is wrong; it needs to be exactly the other way round. It follows that:

- On page 1595, the sentence "Species spatial turnover was higher among urban areas than among rural areas or pairs of urban and rural areas for most taxa." should read: "Species spatial turnover was lower among urban areas than among rural areas or pairs of urban and rural areas for most taxa."

- On pages 1595 and 1596, the sentence "Our results indicate an increasing isolation of species assemblages with urbanisation [...]." should read: "Our results indicate an increasing isolation of species assemblages with increasing distance [...]."

- On page 1600 , "For $\beta$-diversity, the $\beta_{\text {sim }}$ similarity index was calculated from presence-absence tables [...]" should read: "For $\beta$-diversity, the $\beta_{\text {dissim }}$ dissimilarity index was calculated from presence-absence tables [...]." Also, " $\beta_{\text {sim }}=a /(a+\min$ $(b, c))$ " should read " $\beta_{\text {dissim }}=\operatorname{sqrt}(1-(a /(a+\min (b, c))))$ ".

- On pages 1600 and 1601 , the sentences "This index is a measure of similarity taking into account all species that are shared by two areas and the smaller number of species not shared. Its values range from zero to one; the upper limit indicating complete similarity of communities and the lower limit indicating no similarity at all." should read: "This index is a measure of dissimilarity taking into account all species that are shared by two areas and the smaller number of species not shared. Its values range from zero to one; the upper limit indicating complete dissimilarity of communities and the lower limit indicating complete similarity." Also, "Note that an increase in $\beta_{\text {sim }}$ is considered a decrease in $\beta$-diversity." should read: "An increase in $\beta_{\text {dissim }}$ is considered an increase in $\beta$-diversity."

- On page 1603 , for the sentences "In the protected areas within Halle, the $\beta_{\text {sim }}$ similarity index and therefore the similarity of the species assemblages is lowest for butterflies, snails and all plant taxa. It is lowest for carabid beetles and birds in the protected areas within the district of Saalkreis. Pairs of urban and rural areas are more similar than pairs of urban areas for all species groups (Figs. 4 and 5)." " $\beta_{\text {sim }}$ " should be replaced with " $\beta_{\text {dissim" }}$ " "similarity" should be replaced with "dissimilarity".

- In Figures 4 and 5, page 1606, the y-axis label needs to say "beta-dissim dissimilarity index" instead of "beta-sim similarity index". In the figure captions of both figures "Boxplots showing the $\beta_{\text {sim }}$ similarity index [...]." should be: "Boxplots showing the $\beta_{\text {dissim }}$ dissimilarity index [...]."

- The Discussion-paragraph on "Isolation", starting on page 1608 is based on the wrong interpretation of results. Originally, the paragraph reads: "[...] our results indicate stronger isolation mechanisms among urban than among rural protected areas: the $\beta_{\text {sim }}$ similarity index of butterflies, snails, lichens, mosses and vascular plants is lowest among urban protected areas, even lower than among pairs of urban and rural protected areas. This suggests that species mainly move between pairs of rural protected areas and between pairs of urban and rural protected areas, but less between pairs of urban 


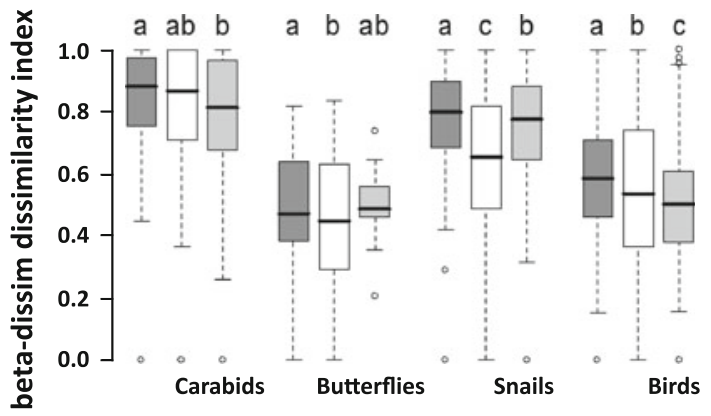

Fig. 4 Boxplots showing the $\beta_{\text {dissim }}$ dissimilarity index for carabid beetles, butterflies, snails and birds for pairs of urban and rural (dark grey bars), urban (white bars) and rural (light grey bars) protected areas (Halle and Saalkreis, Central Germany). The boxplots represent median (line), 25-75\% quartiles (boxes), ranges (whiskers) and extreme values (circles). The letters above the boxplots indicate significant differences between them
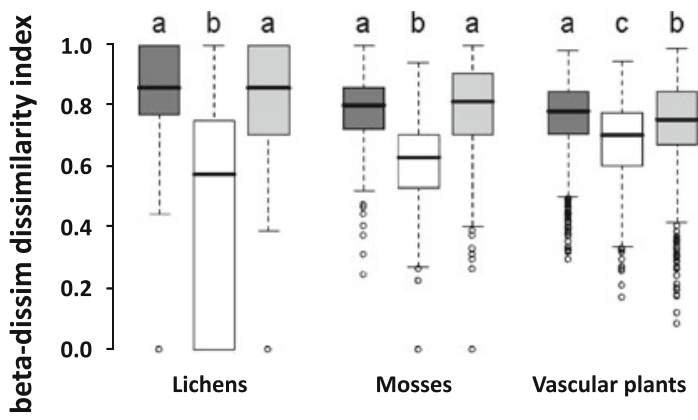

Fig. 5 Boxplots showing the $\beta_{\text {dissim }}$ dissimilarity index for lichens, mosses and vascular plants for pairs of urban and rural (dark grey bars), urban (white bars) and rural (light grey bars) protected areas (Halle and Saalkreis, Central Germany). The boxplots represent median (line), 25-75\% quartiles (boxes), ranges (whiskers) and extreme values (circles). The letters above the boxplots indicate significant differences between them

protected areas. [...] Our results suggest that the type of the landscape matrix surrounding the protected areas plays an important role in the isolation of species assemblages, not distance itself. [...] In summary, we argue that the built-up urban matrix is more resistant to species migrations than the rural matrix and the river valleys. [...] This isolation causes lower $\alpha$-diversity and higher $\beta$-diversity in the urban protected areas. [...]." With the correct interpretation, our results do not indicate stronger isolation mechanisms among urban than among rural protected areas. As the urban protected areas are located closer to each other than the rural protected areas or pairs of urban and rural protected areas, similarity does simply decrease with increasing distance. Species mainly move between pairs of protected areas that are close to each other. To test whether the urban matrix has a stronger isolation effect than the rural matrix, we would need to account for the distance among protected areas when calculating species turnover; i.e. if turnover was higher along, e.g., $100 \mathrm{~m}$ in the city than along $100 \mathrm{~m}$ in the countryside, then our suggestion that the urban matrix has a 
stronger isolation effect than the rural matrix would still be correct. However, we did not test this and cannot draw conclusions regarding this question.

- In the Conclusions, which start on page 1609 the following changes are necessary: "The protected areas in the rural district of Saalkreis [...] had a lower spatial species turnover." This should read: "The protected areas in the rural district of Saalkreis [...] had a higher spatial species turnover." The sentence "This shows that $\beta$-diversity rather than $\alpha$-diversity causes the high species richness generally found in urban areas and points to a higher isolation of species in protected areas within an urban matrix." needs to be deleted. The sentence "If species richness is distributed over a large area (high $\beta$-diversity) more space for protection is needed." should read: "As species richness highly depends on area more space for protection is needed." Moreover, we want to add that with less space being provided for species protection, also isolation among protected areas is likely to increase.

- Also, the following reference is added: Chessel D, Dufour AB, Thioulouse J (2004) The ade4 package-I-One-table methods. R News 4:5-10 